\title{
Large scale use of SARS-CoV-2 antigen-based detection tests: a three-month experience in Guatemala, June-August 2020
}

\author{
Lorena Gobern', Liz Parra², Antonio A. Paredes ${ }^{1}$, Rafael A. León ${ }^{3}$, Jairo Mendez-Rico4, and \\ Marc Rondy ${ }^{2}$
}

Suggested citation Gobern L, Parra L, Paredes AA, Rafael A. León RA, Mendez-Rico J, Rondy M. Large scale use of SARS-CoV-2 antigen-based detection tests: a three-month experience in Guatemala, June-August 2020. Rev Panam Salud Publica. 2020;44:e174. https://doi.org/10.26633/RPSP.2020.174

ABSTRACT Objectives. To measure protocol adherence and antigen-based detection tests (AgDT) negative predictive value after 3 months of massive use as a diagnostic tool for COVID-19 in Guatemala.

Methods. The study period included nasopharyngeal swabs taken between March 12 and August 31, 2020, which results were entered in the national COVID-19 information system. Proportional increase in testing between one month before and one month after the introduction of AgDT (May 9-June 8 vs. June 9-July 8) was measured.

Results. After AgDT introduction, there was a 139\% increase in SARS-CoV-2 testing. Between June 9 and August 31, 7.8\% of 110657 AgDT-negative patients had follow-up RT-PCR testing. Of them, 30\% were RT-PCR positive.

Conclusions. While introducing AgDT improved access to diagnostics, ensuring the availability of timely RT-PCR capacities to confirm diagnosis is also key.

Keywords

Laboratory test; clinical laboratory techniques; reverse transcriptase polymerase chain reaction; coronavirus infections; Guatemala.

Molecular detection by reverse transcription-polymerase chain reaction (RT-PCR) methods is considered the gold standard for diagnosis of acute SARS-CoV-2 infection (1). Despite significant efforts to strengthen diagnostic capacities, many countries, including Guatemala, were overwhelmed by the number of RT-PCR tests required, leading to problematic delays in results delivery. The Pan American Health Organization (PAHO) recently recognized point-of-care antigen-based detection tests (AgDT) as a strategy to decentralize access and complement primary health care level diagnosis capacity in the Americas (2).

\footnotetext{
Ministry of public health and social assistance of Guatemala, Guatemala City, Guatemala

2 Pan American Health Organization, Guatemala City, Guatemala. $\triangle$ Marc

Rondy, rondymar@paho.org
}

In Guatemala, the first case of COVID-19 was detected through Charite based RT-PCR protocol (3) at the national reference laboratory on March 13, 2020. Over the following weeks, machine-based RT-PCR capacities were extended at regional level (GeneXpert (4) and Filmarray (5)). By June, the number of daily RT-PCR tests performed was close to 1000 , positivity was nearly $40 \%$ and delay for results delivery was around one week (6). Meanwhile, a hospital-based validation of Standard Q COVID-19 Ag tests (7) suggested performances similar to what was reported in systematic reviews (8) at $65 \%-84 \%$ sensitivity and $100 \%$ specificity (9). On June 9 , the Ministry of Health

\footnotetext{
3 Sunstone Bioinformatics, Guatemala City, Guatemala

4 Pan American Health Organization, Washington, DC, United States of America
} 
$(\mathrm{MoH})$ updated its surveillance protocol to include AgDT as a complementary diagnostic tool to be used in every setting (10). Various AgDT brands were available and have been used in the country. Per the surveillance protocol, patients with positive AgDT could be classified as confirmed; those with negative AgDT were required to have a second swab taken and tested with RT-PCR to be discarded as non-cases.

The objectives of this analysis were to measure protocol adherence and AgDT negative predictive value after 3 months of massive AgDT use as a diagnostic tool for COVID-19 in Guatemala.

\section{METHODS}

The study period included nasopharyngeal swabs taken between March 12 and August 31, 2020. Suspected COVID-19 case definitions and testing strategies changed throughout the initial 6 months of the outbreak in Guatemala. Before May 28, suspected case definition included clinical and epidemiological contexts and both cases and contacts were tested. Since May 29, suspected COVID-19 cases have been defined as any individual with acute respiratory infection (fever and one respiratory sign). Any suspected case is eligible for testing; asymptomatic contacts are not eligible for testing.

\section{Data collection}

The $\mathrm{MoH}$ requires all laboratories running COVID-19 diagnostic tests to be registered. The $\mathrm{MoH}$ epidemiology department provides registered users a username and password to access the national COVID-19 information system (epiweb). Laboratory data entry clerks register results of all processed samples as well as basic demographic information extracted from a notification form in epiweb.

\section{Data collected}

Data entered in epiweb include laboratory name; patient's age, gender, and place of residence; type of test used; and the dates of symptoms onset, sample collection and results delivery.

\section{Data analysis}

To create a database matching AgDT and RT-PCR tests, records of the same individual were identified based on name, age, sex, and swabbing date (+/- 3 days). Laboratories were classified as from $\mathrm{MoH}$, social security (branch of the Guatemalan Ministry of Work and Social Provision that provides health services to salaried employees) or private services. Patients were defined as symptomatic if the date of symptoms onset was not missing.

Proportional increase in testing between one month before and one month after the introduction of AgDT (May 9-June 8 vs. June 9-July 8) was measured. Changes in testing trend during weekdays of the month preceding and the month following the introduction of AgDT were compared using a Chow Test comparing slopes (11).

Median delivery delay between swabbing and results delivery date were compared using Mood's median test.

In the period June 9-August 31, proportion of follow-up RT-PCR and proportion of RT-PCR-positive tests among
AgDT-negative patient-groups were compared using t-test. To estimate the number of false negatives without follow-up PCR, we calculated strata specific estimates crossing gender, age group, presence of symptoms, laboratory type and place of residence and summed the obtained data.

All analysis was conducted using $\mathrm{R}$ statistical package (R Foundation for Statistical Computing, Vienna, Austria) (12).

This study did not require approval by an ethics committee as it was in the context of public health surveillance. All data was handled under confidentiality protection.

\section{RESULTS}

Between March 12 and August 31, 2020, 248098 SARS-CoV-2 tests were reported in Guatemala, of which $31 \%$ were positive; of $233715(94 \%)$ registers with information regarding the test used, 82373 (35\%) were tested with RT-PCR and 151342 (65\%) with AgDT (Figure 1).

Laboratory diagnostics increased by 139\% (daily average of 1152 vs. 2749 screened individuals) between the month before and the month after AgDT introduction (increase of 220\% inside and $58 \%$ outside the capital city department) and the testing trend significantly increased between the two periods (Chow test $\mathrm{p}$-value $=0.02$ ) (Figure 2). Median delay for results delivery was 3 days before and 4 days after AgDT introduction $(\mathrm{p}<0.01)$. After AgDT introduction, median delay for results delivery was 2 days for AgDT and 21 days for RT-PCR ( $p<0.01$ ). Among individuals who had a follow-up PCR test, median delays were one day between first swab and negative AgDT result, two days between negative AgDT result and second swab and three days between second swab and RT-PCR result.

The overall positivity for RT-PCR and AgDT tests was 36.0\% and $26.9 \%$ respectively. Of 109657 swabs negative by AgDT, 8 $595(7.8 \%)$ had a registered result for RT-PCR (Table 1$)$. The proportion of follow-up RT-PCR tests in negative AgDT was 7\% in patients under 60 years of age and $14 \%$ in those aged 60 years and above; it was $27 \%, 4 \%$, and $3 \%$ among $\mathrm{MoH}$, social security, and private laboratories, respectively.

Of 8595 AgDT-negative swabs with RT-PCR results, 6 $025(70 \%)$ tested negative by RT-PCR; $72 \%, 56 \%$, and $70 \%$ in recently symptomatic, symptomatic for 10 days or more, and asymptomatic patients, respectively. It was $68 \%, 59 \%$, and $81 \%$ in $\mathrm{MoH}$, social security, and private laboratories, respectively.

Of 102062 AgDT-negative swabs without RT-PCR results, we estimated that 37339 (95\% confidence interval: 26 560-48 118) would have tested positive in RT-PCR.

\section{DISCUSSION}

AgDT introduction in Guatemala led to a significant increase in SARS-CoV-2 testing trends, ultimately increasing the sensitivity and timeliness of surveillance activities. However, only $7.8 \%$ of AgDT-negative patients were tested with RT-PCR and, of those, 30\% were positive.

The increase in SARS-CoV-2 tests after June 9 cannot be fully attributed to AgDT introduction. Ideally, changes in COVID19 incidence in the population should be assessed in order to isolate the true impact of AgDT in access to diagnosis. In the absence of these data, comparison of slopes of testing curves between the two periods demonstrated a significant increase in testing trends after AgDT introduction. Overall results delivery 


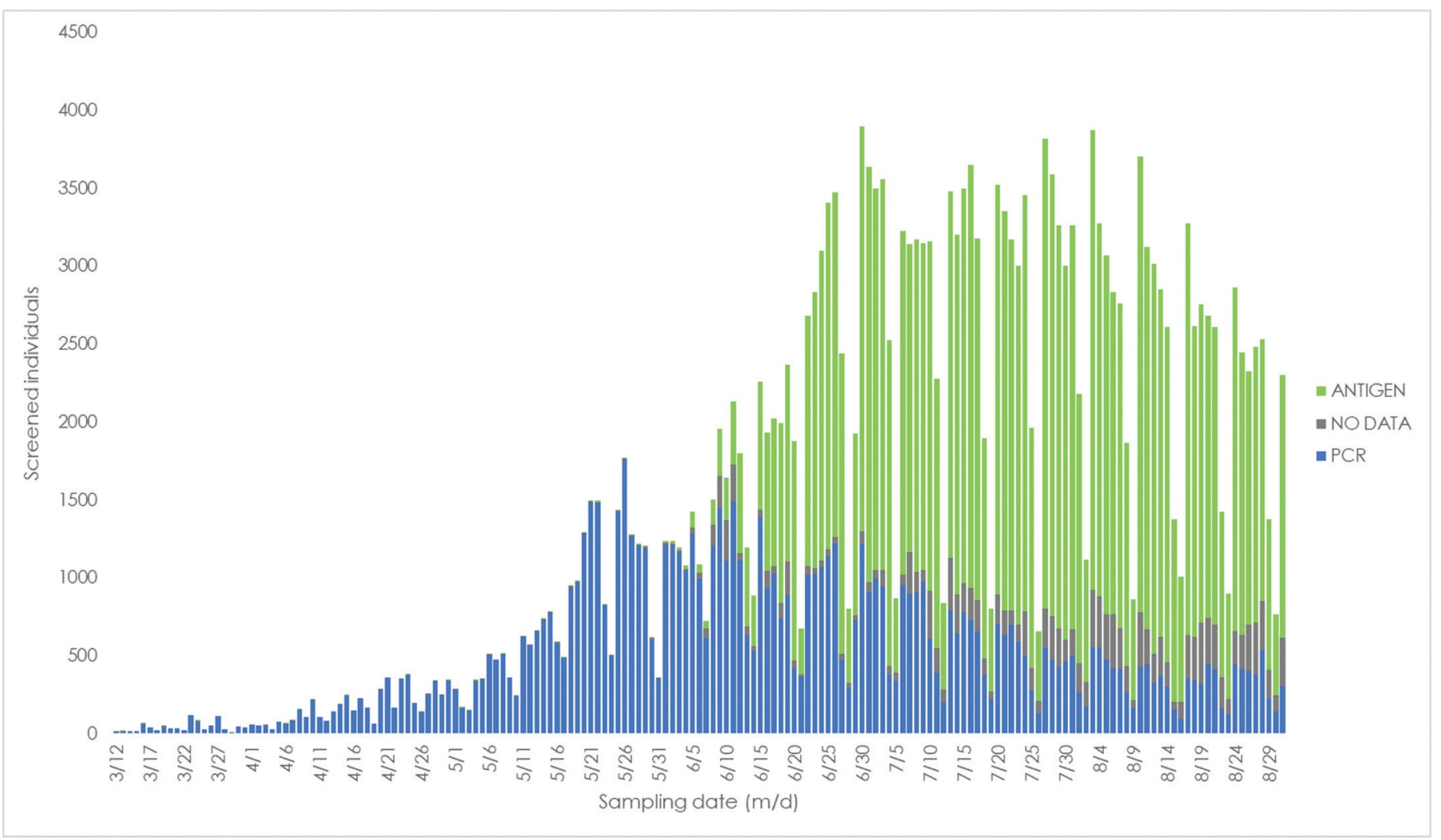

FIGURE 2. Laboratory diagnosis increase after AgDT introduction, Guatemala, 2020

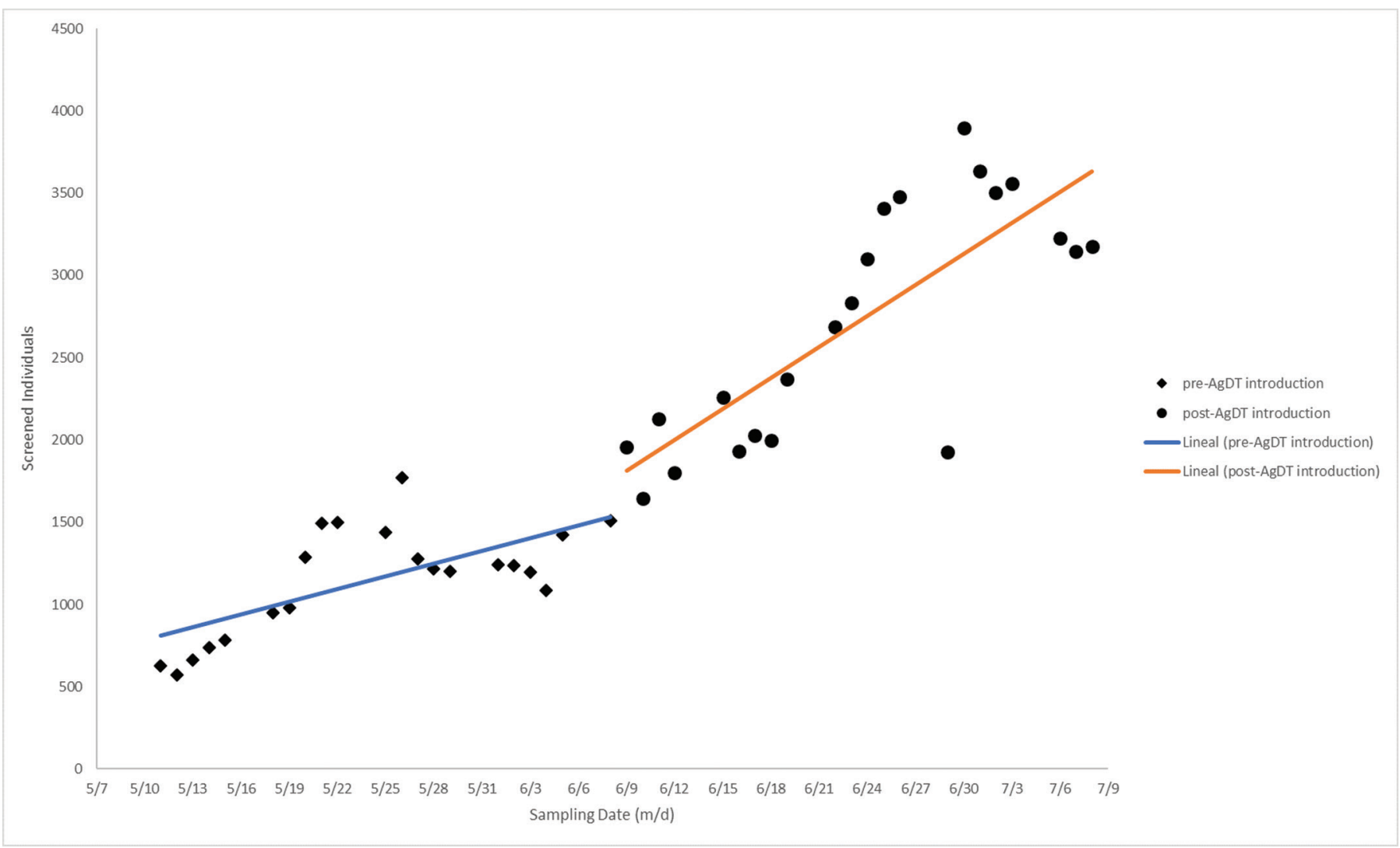


TABLE 1. Patients' profile, laboratory type, department of resident and COVID-19 positivity percentage, Guatemala, 2020

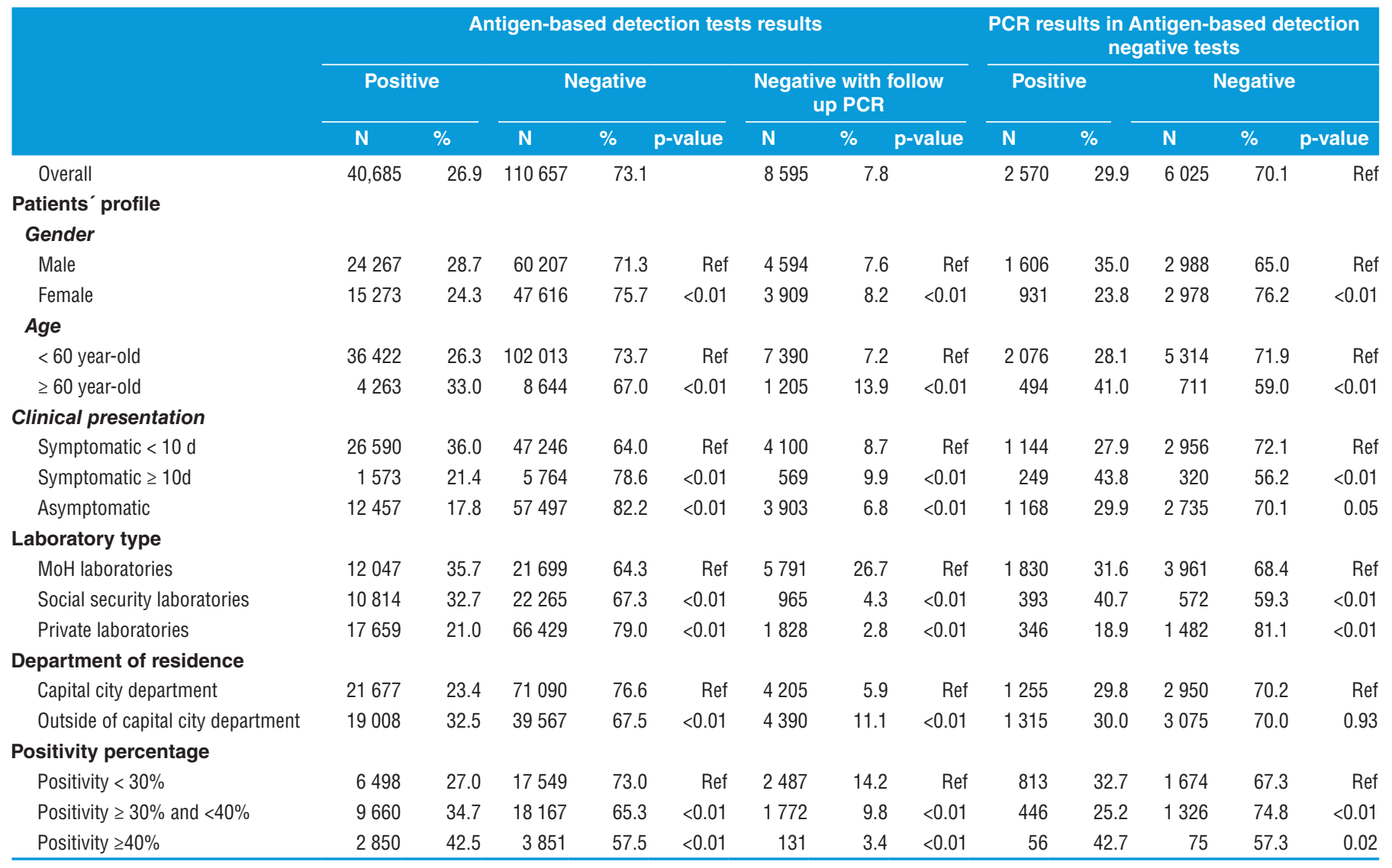

delays did not decrease after the introduction of AgDT. However, longer results delivery delays after AgDT introduction were fully attributable to RT-PCR tests, for which results delivery increased from 3 to 21 days between the two time periods. AgDT introduction allowed maintaining a reasonable diagnostic turn-around-time at a time when RT-PCR availability became more scarce.

Based on our results, $\mathrm{MoH}$ health services were the most compliant $(26.7 \%)$ and private practices the least compliant $(2.8 \%)$ with the MoH guidelines of running systematic RT-PCR on AgDT-negative patients. In private laboratories, as of August 31, the average price of an RT-PCR test was between three and ten times higher than for an AgDT. It is likely that individual economical resources were the main reason for not following the $\mathrm{MoH}$ protocols in private laboratories. Lack of adherence to the protocol from $\mathrm{MoH}$ and social security services could be associated with unacceptable delay and complicated logistics in the RT-PCR testing process.

Overall, the negative predictive value (NPV) was $70 \%$, meaning that almost one-third of AgDT-negative tests were false negatives. NPV was the lowest among patients sampled more than nine days after symptoms onset, highlighting the importance of using AgDT in the first days after symptoms onset $(2,5)$ in suspected cases. Since NPV is negatively associated with prevalence (14), its value was highest in private hospitals, where only $21 \%$ of AgDT results were positive, and lowest in the departments of Guatemala that had an overall positivity proportion of $40 \%$ and above. The risks of false negative AgDT results (and low NPV) are higher in high incidence settings.

Considering strata specific NPV we estimated that, between June 9 and August 31, more than 37000 COVID-19 cases received a negative AgDT results without follow-up RT-PCR. Among negative AgDT individuals with a subsequent RT-PCR tests, the median delay between negative AgDT result and positive RT-PCR results was five days. Considering that SARSCoV-2 RNA highest viral loads are observed around the day of symptom onset and follow a gradual decline over time (15), reducing this period, during which an infected individual is wrongly considered as free from SARS-CoV2, is critical. Indeed, false negative AgDT results could lead to a false sense of security, low adherence to isolation measures, and greater probability of infecting others (16). Such consequences may be of particular concern for individuals with severe symptoms, healthcare workers, and people living in closed settings. While our data suggest a proportion of RT-PCR follow-up two times higher in older adults ( $14 \%$ vs. $7 \%$ ), it remains an uncommon practice in that population, and confirmatory testing should be increased.

Being based on surveillance data, this work is affected by some limitations. We cannot exclude that the matching process to combine AgDT and RT-PCR laboratory results, subject to data entry error, may have underestimated the proportion of follow-up RT-PCR tests. However, this underestimation is 
likely to be minimal and non-differential between comparison groups.

Cases were defined as symptomatic based on the documentation of a date of symptom onset. Missing dates may have led to misclassifying a number of symptomatic cases as asymptomatic; this could explain the high number of asymptomatic patients in our analysis despite their theorical per-protocol exclusion from testing. However, the observed lower proportion of positive AgDT results and relatively high NPV are concordant with a high proportion of asymptomatic persons in that group.

While more than 20 AgDT brands were gradually available in the country, Standard Q COVID-19 Ag tests (7) were the most commonly used during the period of analysis (personal communication). However, since AgDT brand was not recorded in the information system, we could not stratify results per brand. An increasing number of AgDT diagnostic tests are being made available and their formal evaluation tracked as part of the Access to COVID-19 Tools (ACT) Accelerator global collaboration (17). Measuring and reporting on their field performance is critical to refine recommendations towards better performing tools.

Finally, due to $\mathrm{MoH}$ guidelines recommending follow-up RT-PCR for negative AgDT only, we could not compute its positive predictive value. In settings with low prevalence of positives (contention phase), false positives may become a concerning issue and RT-PCR confirmation tests may be recommended for specific situations (13).

Considering the high proportion of false negatives and lack of adherence to the diagnostic algorithm, the Guatemalan $\mathrm{MoH}$ published updated guidelines recommending that
AgDT-negative suspected cases without follow-up RT-PCR tests should remain isolated until clinical recovery (18). In its latest interim guidance, the World Health Organization mostly recommends AgDT use to confirm or respond to COVID-19 outbreaks in remote settings, institutions and semi-closed communities where nucleic acid amplification test is not immediately available and to monitor disease incidence in communities with widespread transmission (19).

\section{CONCLUSION}

Including AgDT in the Guatemalan testing strategy led to a clear improvement in access to diagnostics by the population. To successfully implement this strategy, ensuring the availability of timely RT-PCR capacities to confirm negative diagnosis in high incidence settings and positive diagnosis in low incidence settings is key.

Acknowledgments. To all health professionals who are facing COVID-19; to Edwin Asturias and the Presidential commission against coronavirus (CopreCOVID) for promoting the large-scale use of SARS-CoV-2 antigen-based detection tests in Guatemala; to David Little and Emily Zielinski Gutierrez for their advices and manuscript revision.

\section{Conflict of interests. None declared.}

Disclaimer. Authors hold sole responsibility for the views expressed in the manuscript, which may not necessarily reflect the opinion or policy of the RPSP/PAJPH and/or PAHO.

\section{REFERENCES}

1. World Health Organization. Laboratory testing for coronavirus disease 2019 (COVID-19) in suspected human cases: interim guidance, 2 March 2020. World Health Organization. https://apps.who.int/ iris/handle/10665/331329.

2. Pan American Health Organization (PAHO) / World Health Organization (WHO). Expansion of the COVID-19 diagnostic laboratory network: Implementation of antigen-based detection tests; 2020.

3. Corman VM, Landt O, Kaiser M, Molenkamp R, Meijer A, Chu DK, et al. Detection of 2019 novel coronavirus (2019-nCoV) by real-time RT-PCR. Euro Surveill. 2020;25(3).

4. US Food and Drug Administration. Xpert Xpress SARS-CoV-2. [Internet]. [cited 26 October 2020]. Available from: https://www. fda.gov/media/136314/download

5. US Food and Drug Administration. BioFire ${ }^{\circledR}$ COVID-19 Test [Internet]. [cited 26 October 2020]. Available from: https://www.fda. gov/media/136353/download

6. Tablero de situación epidemiologica de COVID-19 en Guatemala. Ministry of public health and social assistance of Guatemala, Guatemala. [Internet]. [cited 17 October 2020]. Available from: https:// tablerocovid.mspas.gob.gt/

7. SD Biosensor. STANDARD Q COVID-19 Ag: product description. [Internet]. [cited 24 October 2020]. Available from: http:/ / sdbiosensor.com/xe/product/7672

8. Dinnes J, Deeks JJ, Adriano A, Berhane S, Davenport C, Dittrich S, et al. Rapid, point-of-care antigen and molecular-based tests for diagnosis of SARS-CoV-2 infection. Cochrane Infectious Diseases Group, editor. Cochrane Database of Systematic Reviews [Internet]. 26 August 2020 [cited 15 October 2020]. Available from: http:/ / doi. wiley.com/10.1002/14651858.CD013705
9. Ministry of public health and social assistance of Guatemala, Presidential commission against coronavirus (CopreCOVID), Guatemala. Guía nacional para la realización de pruebas diagnósticas para COVID-19; 2020.

10. Ministry of public health and social assistance of Guatemala. Procedures for COVID-19 preparedness and response. Epidemiological surveillance of acute respiratory infection by covid-19: interim guidance, 9 June 2020 [Internet]. Guatemala; 2020. Available from: http://epidemiologia.mspas.gob.gt/informacion/ coronavirus-2019-ncov/descargas-coronavirus-covid-19.

11. Chow GC. Tests of Equality Between Sets of Coefficients in Two Linear Regressions. Econometrica. 1960;28(3):591.

12. R Core Team. R: A language and environment for statistical computing. R Foundation for Statistical Computing, Vienna, Austria. Available from: https://www.R-project.org/.

13. Centers for Disease Control and Prevention. Interim Guidance for Rapid Antigen Testing for SARS-CoV-2. Updated Sept 4, 2020. [Internet]. [cited 7 September 2020]. Available from: https://www.cdc. gov/coronavirus/2019-ncov/lab/resources/antigen-tests-guidelines.html

14. Altman DG, Bland JM. Diagnostic tests 2: Predictive values. BMJ. 1994;309(6947):102.

15. World Health Organization. Transmission of SARS-CoV-2: implications for infection prevention precautions: Scientific brief, 9 July 2020. En [cited 26 October 2020]. Available from: https://apps.who. int/iris/handle/10665/333114

16. Woloshin S, Patel N, Kesselheim AS. False Negative Tests for SARSCoV-2 Infection - Challenges and Implications. N Engl J Med. 2020;383(6):e38. 
17. Find evaluation of SARS-COV-2 antigen detecting tests. [Internet]. [cited 15 October 2020]. Available from: https://www.finddx.org/ covid-19/sarscov2-eval-antigen/

18. Ministry of public health and social assistance of Guatemala. Procedures for COVID-19 preparedness and response. Epidemiological surveillance of acute respiratory infection by covid-19: interim guidance, 24 August 2020 [Internet]. Guatemala; 2020. Available from: http://epidemiologia.mspas.gob. gt/informacion/coronavirus-2019-ncov/descargas-coronaviruscovid-19.
19. World Health Organization. Antigen-detection in the diagnosis of SARS-CoV-2 infection using rapid immunoassays: Interim guidance, 11 September 2020. https:/ /apps.who.int/iris/handle/10665/334253.

Manuscript submitted on 22 September 2020; revised version accepted for publication on 4 November 2020.

\section{Empleo a gran escala de pruebas de detección de SARS-CoV-2 basadas en antígeno: experiencia de tres meses en Guatemala, junio-agosto de 2020}

RESUMEN

Objetivos. Evaluar la adherencia al protocolo y el valor predictivo negativo de las pruebas de detección basadas en antígeno (AgDT) después de 3 meses de uso masivo como método diagnóstico para la COVID-19 en Guatemala.

Métodos. Se estudiaron hisopados nasofaríngeos tomados entre el 12 de marzo y el 31 de agosto de 2020, cuyos resultados constaban en el sistema de información nacional de COVID-19. Se midió el aumento proporcional del número de pruebas entre un mes antes y un mes después de la introducción de las AgDT (9 de mayo a 8 de junio, frente a 9 de junio a 8 de julio).

Resultados. Después de la introducción de AgDT hubo un aumento del 139\% en el número de pruebas de SARS-CoV-2. Entre el 9 de junio y el 31 de agosto, el 7,8\% de 110657 pacientes negativos según una AgDT se sometieron a una prueba de seguimiento con RT-PCR. De ellos, el 30\% presentó una RT-PCR positiva.

Conclusiones. Aunque la introducción de AgDT mejoró el acceso al diagnóstico, también es clave asegurar la disponibilidad oportuna de RT-PCR para confirmar el diagnóstico.

Palabras clave Prueba de laboratorio; técnicas de laboratorio clínico; reacción en cadena de la polimerasa de transcriptasa inversa; infecciones por coronavirus; Guatemala. 